\title{
ON TENSOR PRODUCTS AND EXTENDED CENTROIDS ${ }^{1}$
}

\author{
W. K. NICHOLSON AND J. F. WATTERS ${ }^{2}$
}

\begin{abstract}
For prime algebras $R$ and $S$ over a field $F$ it is shown that each nonzero ideal of $R \otimes S$ contains a nonzero element $r \otimes s, r \in R, s \in S$, if and only if $C(R) \otimes C(S)$ is a field, where $C(R)$ (respectively $C(S)$ ) is the extended centroid of $R$ (respectively $S$ ).
\end{abstract}

A prime $F$-algebra $R$ is said to be closed (or centrally closed) if $F$ coincides with the extended centroid of $R$. This terminology arises in [2] where it is noted [2, Remark p. 60] that if $R$ is a closed prime unital $F$-algebra and $S$ is any unital $F$-algebra, then any nonzero ideal of $R \otimes_{F} S$ contains a nonzero element of the form $r \otimes s, r \in R, s \in S$. The aim of this note is to show that this condition is satisfied if and only if $C(R) \otimes_{F} C(S)$ is a field, where $C(R)$ and $C(S)$ are the extended centroids of the prime algebras $R$ and $S$, respectively, which are not necessarily unital. Observe that when $R$ is closed $C(R)=F$ and $C(R) \otimes_{F} C(S)=C(S)$ is a field. In addition to the notation already introduced we write $R^{\prime}=R C(R)$ for the central closure of $R$. All tensor products will be over $F$ so the subscript $F$ will be omitted. To deal with not necessarily unital rings note that a prime $F$-algebra $R$ can be embedded as an ideal in a prime $F$-algebra $R^{\#}$ with unity and so $C(R)=C\left(R^{\#}\right)$. Throughout this paper $R$ (and $S$ ) are prime associative algebras so $C(R)$ is a field.

Lemma 1 [4, TheOREM 1]. Let $x, y \in R^{\prime}$ such that $x r y=y r x$ for all $r \in R$. Then $x$ and $y$ are $C(R)$-dependent.

Lemma 2. If $\mu(x \otimes y)=0$ for $x \in R^{\prime}, y \in S^{\prime}$ and $\mu \in C(R) \otimes C(S)$, then either $\mu=0$ or $x \otimes y=0$.

PRoof. If $\mu \neq 0$ write $\mu=\sum_{i=1}^{m} c_{i} \otimes d_{i}$ where $\left\{c_{i}\right\}$ is an $F$-independent set. If $x \neq 0$ then $\left\{c_{i} x\right\}$ is also $F$-independent (since $C(R)$ is a field) so $\mu(x \otimes y)=0$ implies $d_{i} y=0$ for all $i$, whence $y=0$ and $x \otimes y=0$.

THEOREM. Let $R$ and $S$ be prime $F$-algebras. Then the following are equivalent:

(1) $C(R) \otimes C(S)$ is a field;

(2) each nonzero ideal of $R \otimes S$ contains an element $r \otimes s \neq 0, r \in R, s \in S$.

Proof. We first prove the result when $R$ and $S$ are unital.

Received by the editors June 1, 1982. Presented to the A.M.S. on April 29-30, 1983.

1980 Mathematics Subject Classification. Primary 16A12, 16A20; Secondary 15A69.

'Supported in part by NSERC Grant A8075.

${ }^{2}$ Supported in part by a grant from the University of Leicester Research Board. 
(1) $\Rightarrow$ (2). Let $0 \neq I \triangleleft R \otimes S$ and let $0 \neq x=\sum_{i=1}^{m} r_{i} \otimes s_{i} \in I$ where $m$ is minimal among all such representations of nonzero elements of $I$. Then $\left\{s_{i}\right\}$ is $F$-independent and, for all $r \in R, y=x\left(r r_{1} \otimes 1\right)-\left(r_{1} r \otimes 1\right) x \in I$. The minimality of $m$ forces $y=0$ and so $r_{i} r r_{1}=r_{1} r r_{i}$ for all $i$. Since $r_{1}$ and $r_{i}$ are nonzero $r_{i}=c_{i} r_{1}$ for some $c_{i} \in C(R)$ by Lemma 1. Similarly $s_{i}=d_{i} s_{1}$ for some $d_{i} \in C(S)$. Put $\lambda=\sum_{i=1}^{m} c_{i} \otimes d_{i}$ $\in C(R) \otimes C(S)$. Thus $x=\lambda\left(r_{1} \otimes s_{1}\right)$ and $\lambda \neq 0$. By $(1) r_{1} \otimes s_{1}=\mu x$ for $\mu=\lambda^{-1} \in$ $C(R) \otimes C(S)$.

Write $\mu=\sum_{j=1}^{n} e_{j} \otimes f_{j}$ with $e_{j} \in C(R), f_{j} \in C(S)$. From the construction of $R^{\prime}$ and $S^{\prime}$ there are nonzero ideals $A$ of $R, B$ of $S$ such that $A e_{j} \subseteq R$ and $B f_{j} \subseteq S$ for all $j$. Hence $(A \otimes B) \mu \subseteq R \otimes S$ and $(A \otimes B)\left(r_{1} \otimes s_{1}\right) \subseteq I$. Since $R$ and $S$ are prime we have $0 \neq r \in A r_{1}$ and $0 \neq s \in B s_{1}$ so that $r \otimes s \in I$.

$(2) \Rightarrow(1)$. Let $0 \neq \mu \in C(R) \otimes C(S)$. As above we have $0 \neq A \triangleleft R, 0 \neq B \triangleleft S$ and $\mu(A \otimes B)=(A \otimes B) \mu=I \triangleleft R \otimes S$. From Lemma $2, I \neq 0$ and so $I$ contains a length one tensor from (2). Select $\sum_{i=1}^{m} a_{i} \otimes b_{i} \in A \otimes B$ with $m$ as small as possible so that $\mu\left(\sum a_{i} \otimes b_{i}\right)$ is of length one, say $\mu\left(\sum a_{i} \otimes b_{i}\right)=r_{1} \otimes s_{1} \neq 0, r_{1} \in R, s_{1} \in S$. Then, for all $r \in R$ and $i=1,2, \ldots, m,\left(a_{i} r \otimes 1\right)\left(r_{1} \otimes s_{1}\right)-\left(r_{1} \otimes s_{1}\right)\left(r a_{i} \otimes 1\right) \in I$. The minimality of $m$ along with Lemma 1 gives $a_{i}=p_{i} r_{1}, p_{i} \in C(R)$. Similarly $b_{i}=q_{i} s_{1}$ for $q_{i} \in C(S)$. With $\lambda=\Sigma p_{i} \otimes q_{i} \in C(R) \otimes C(S)$ we have

$$
(1-\mu \lambda)\left(r_{1} \otimes s_{1}\right)=0 .
$$

By Lemma $2,1=\mu \lambda$ and $C(R) \otimes C(S)$ is a field.

To deal with the not necessarily unital case we use the algebras $R^{\#}$ and $S^{\#}$.

$(1) \Rightarrow(2)$. If $0 \neq I \triangleleft R \otimes S$, then $0 \neq\left(R^{\#} \otimes S^{\#}\right) I\left(R^{\#} \otimes S^{\#}\right)=J \triangleleft R^{\#} \otimes S^{\#}$ and $J \subseteq I$. Now $J$ contains an element $r^{\prime} \otimes s^{\prime} \neq 0$ with $r^{\prime} \in R^{\#}$ and $s^{\prime} \in S^{\#}$. Since $\left(r^{\prime} \otimes s^{\prime}\right)(R \otimes S) \subseteq I$ and $R$ and $S$ are prime, we have $0 \neq r \otimes s \in I$ for some $r \in R, s \in S$.

$(2) \Rightarrow(1)$. It is clear that condition (2) implies that $R \otimes S$ is prime. Hence Theorem 2.3 of [3] implies that $R^{\#} \otimes S^{\#}$ is prime. If $0 \neq J \triangleleft R^{\#} \otimes S^{\#}$ then $0 \neq J(R \otimes S)=I \triangleleft R \otimes S$ contains a length one tensor $r \otimes s, r \in R, s \in S$. Thus $R^{\#} \otimes S^{\#}$ satisfies (2) and $C(R) \otimes C(S)$ is a field.

REMARK. Condition (1) of Theorem 1 is equivalent to the statement that $C(R) \otimes$ $C(S)$ is a domain and $C(R) \otimes C(S)=C(R \otimes S)$. If these properties are possessed by $C(R) \otimes C(S)$ then $R \otimes S$ is prime by [3, Theorem 2.3] and $C(R \otimes S)$ is a field, whence $C(R) \otimes C(S)$ is a field. For the converse we appeal to a recent theorem of Matczuk [5] which says that if $R \otimes S$ is semiprime, then

$$
C(R \otimes S)=C(C(R) \otimes C(S))
$$

(here the extended centroid is defined as in the more general setting of Amitsur [1]). Thus if $C(R) \otimes C(S)$ is a field we have $C(R \otimes S)=C(R) \otimes C(S)$.

COROLlaRY 1. If $R$ and $S$ are simple F-algebras such that $C(R) \otimes C(S)$ is a field, then $R \otimes S$ is a simple F-algebra.

Resco [6] has shown that if $R$ is a closed primitive $F$-algebra and $S$ is a primitive $F$-algebra, then $R \otimes S$ is primitive. His proof depends on property (2) and so can be easily modified to prove the next corollary. 
COROllaRY 2. If $R$ and $S$ are primitive F-algebras such that $C(R) \otimes C(S)$ is a field, then $R \otimes S$ is primitive.

Proof. If $R$ and $S$ are unital then the argument from [6] applies. Otherwise we take the rings $R^{\#}$ and $S^{\#}$, which are now primitive, and then $R^{\#} \otimes S^{\#}$ is primitive and so $R \otimes S$ is primitive.

It is clear that if the hypotheses of Corollary 2 hold then $R^{\prime} \otimes S^{\prime}$ is primitive. However this conclusion follows from $R \otimes S$ being primitive, as we show in the next proposition, which extends the known result that if $R$ is primitive then so is $R^{\prime}$.

Proposition. Let $R$ and $S$ be prime F-algebras such that $R \otimes S$ is primitive. Then $R^{\prime} \otimes S^{\prime}$ is primitive.

Proof. Assume first that $R$ and $S$ are unital. Let $L$ be a left ideal of $R \otimes S$ such that $(R \otimes S) / L$ is a faithful irreducible $R \otimes S$-module. Put $L^{\prime}=\left(R^{\prime} \otimes S^{\prime}\right) L$.

If $1=\Sigma \lambda_{i} l_{i}, \lambda_{i} \in R^{\prime} \otimes S^{\prime}, l_{i} \in L$, then there are $0 \neq A \triangleleft R, 0 \neq B \triangleleft S$ such that $(A \otimes B) \lambda_{i} \subseteq R \otimes S$ and so $0 \neq A \otimes B \subseteq L$. But $(R \otimes S) / L$ is faithful so we have a contradiction and $L^{\prime} \neq R^{\prime} \otimes S^{\prime}$.

Since $R \otimes S$ is prime, $R^{\prime} \otimes S^{\prime}$ is prime by [3, Theorem 2.3]. If $0 \neq X^{\prime} \triangleleft R^{\prime} \otimes S^{\prime}$ and $0 \neq x^{\prime} \in X^{\prime}$, then there are ideals $0 \neq I \triangleleft R, 0 \neq J \triangleleft S$ such that $(I \otimes J) x^{\prime} \subseteq$ $R \otimes S$. Now $0 \neq(C(R) \otimes C(S))(I \otimes J) \triangleleft R^{\prime} \otimes S^{\prime}$, so the primeness of $R^{\prime} \otimes S^{\prime}$ implies that $(I \otimes J) x^{\prime} \neq 0$. Thus $X=(R \otimes S) \cap X^{\prime} \neq 0$ and $X \triangleleft R \otimes S$. Now $L+X=R \otimes S$ so $L^{\prime}+\left(R^{\prime} \otimes S^{\prime}\right) X=R^{\prime} \otimes S^{\prime}$. Hence $L^{\prime}+X^{\prime}=R^{\prime} \otimes S^{\prime}$. Therefore, by the comaximality criterion for primitivity, we have that $R^{\prime} \otimes S^{\prime}$ is primitive.

In the general case the algebra $R^{\#} \otimes S^{\#}$ is prime from [3, Theorem 2.3], and so primitive since it contains $R \otimes S$ as an ideal. Thus $\left(R^{\#}\right)^{\prime} \otimes\left(S^{\#}\right)^{\prime}$ is primitive. However $R^{\prime} \triangleleft\left(R^{\#}\right)^{\prime}$ and $S^{\prime} \triangleleft\left(S^{\#}\right)^{\prime}$ so $R^{\prime} \otimes S^{\prime}$ is primitive.

\section{REFERENCES}

1. S. A. Amitsur, On rings of quotients, Symposia Mathematica 8 (1972), 149-164.

2. T. S. Erickson, W. S. Martindale III and J. M. Osborn, Prime nonassociative algebras, Pacific J. Math. 60 (1975), 49-63.

3. J. Krempa, On semisimplicity of tensor products, (Proc. Antwerp Conf., 1978), Pure and Appl. Math., vol. 51, Dekker, New York, 1979, pp. 105-122.

4. W. S. Martindale III, Prime rings satisfying a generalized polynomial identity, J. Algebra 12 (1969), $576-584$.

5. J. Matczuk, Central closure of semiprime tensor products, Comm. Algebra 10 (1982), 263-278.

6. R. Resco, A reduction theorem for the primitivity of tensor product, Math. Z. 170 (1980), 65-76.

Department of Mathematics and Statistics, The University of Calgary, Calgary, Alberta, CANADA T2N 1N4

DEPARTMENT OF MATHEMATICS, UNIVERSity OF LeICESTER, LEICESTER, ENGLAND LE1 7RH 